

\title{
INCIDENCIA DE LAS ESTRATEGIAS PASIVAS DE DISEÑO ARQUITECTÓNICO EN LA ETIQUETA DE EFICIENCIA ENERGÉTICA EN ARGENTINA
}

\section{IMPACT OF PASSIVE ARCHITECTURAL DESIGN STRATEGIES ON THE ENERGY EFFICIENCY LABEL IN ARGENTINA}

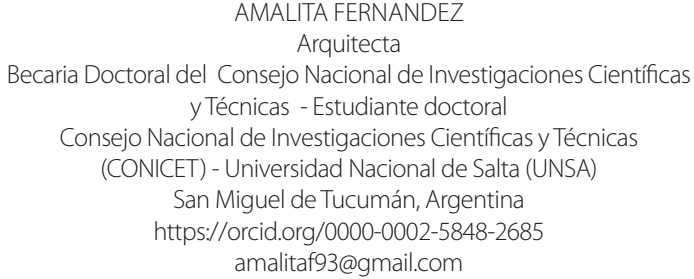

\author{
BEATRIZ SILVIA GARZÓN \\ Doctora en Ciencias \\ Investigadora Independiente CONICET y Profesora Asociada \\ Facultad de Arquitectura y Urbanismo \\ Universidad Nacional de Tucumán - Consejo Nacional de \\ Investigaciones Científicas y Técnicas (CONICET) \\ San Miguel de Tucumán, Argentina \\ https://orcid.org/0000-0003-3130-8895 \\ bgarzon06@gmail.com
}

\author{
DAVID ELSINGER \\ Arquitecto \\ Becario Doctoral del Consejo Nacional de Investigaciones Científicas \\ y Técnicas - Estudiante doctoral Facultad de Arquitectura y Urbanismo \\ Universidad Nacional de Tucumán - Consejo Nacional de \\ Investigaciones Científicas y Técnicas (CONICET) \\ San Miguel de Tucumán, Argentina \\ https://orcid.org/0000-0003-1166-7575 \\ delsinger@herrera.unt.edu.ar
}

\section{RESUMEN}

El trabajo presenta un análisis comparativo realizado entre el Índice de Prestaciones Energéticas (IPE), definido por el Programa Nacional de Etiquetado de Viviendas basándose en la Norma IRAM 11900, y las Estrategias Pasivas de Diseño Arquitectónico (EPDA) definidas en dicha norma. La comparación es aplicada a soluciones de rehabilitación energética para la evaluación de mejoras en Eficiencia Energética (EE) en una vivienda en San Miguel de Tucumán, Argentina. Tiene como objetivo evaluar la incidencia de las EPDA en el valor de IPE de la vivienda. Se realiza una valoración con respecto a la importancia que tienen las mismas como punto de partida para lograr una arquitectura más confortable, más eficiente desde el punto de vista energético y más sustentable. Se concluye que las estrategias pasivas no influyen sustancialmente de manera numérica en el IPE el cual determina la etiqueta de eficiencia energética de unidades residenciales en Argentina.

Palabras clave

arquitectura bioclimática, vivienda, renovación arquitectónica

\section{ABSTRACT}

This work proposes making a comparative analysis between the Energy Supply Index (IPE, in Spanish), defined by the National Housing Labeling Program based on the IRAM 11900 Standard, and the Passive Architectural Design Strategies (EPDA, in Spanish) defined in said standard. The comparison is applied to energy retrofit solutions for the evaluation of the improvements in Energy Efficiency (EE) in a dwelling in San Miguel de Tucumán, Argentina. Its goal is to evaluate the incidence of EPDAs on the IPE value of the dwelling. An assessment is made regarding their importance as a starting point for achieving a more comfortable, more energy efficient and sustainable architecture. It is concluded that passive strategies do not have a numerically substantial influence on the IPE, that determines the energy efficiency label of residential units in Argentina. 


\section{INTRODUCCIÓN}

La reducción del impacto ambiental es una preocupación mundial que ha despertado el compromiso de muchos gobiernos para direccionar sus leyes en función del desarrollo sostenible (Aragón, de Olivera Pamplona y Medina, 2012). La problemática ambiental global conduce a que numerosos países incorporen políticas de Eficiencia Energética (EE) con el fin de disminuir el consumo de energía, aspecto que puede observarse en medidas como etiquetados o pasaportes de EE (AlonsoFrank y Kuchen, 2017). El sector residencial consume a nivel global una cantidad significativa de energía, por tal motivo resulta fundamental desarrollar e implementar sistemas arquitectónicos bioclimáticos que contribuyan a la disminución del consumo energético (Manzano, Montoya, Sabio-Ortega, y García-Cruz, 2015). Se trata, asimismo, de reducir el consumo sin afectar el confort térmico edilicio. En Argentina, para contribuir a lograr la meta nacional de ahorro energético, es esencial la implementación de medidas de EE en el sector residencial, ya que este representa el $27 \%$ del consumo total del país. Además, este sector puede ser abordado desde múltiples enfoques, lo que le otorga un gran potencial de mejora (Ministerio de Desarrollo Productivo, 2017).

Los esquemas de certificación energética para edificios surgieron a principios de la década de 1990 como método esencial para mejorar la EE, minimizando el consumo de energía y permitiendo mayor transparencia con respecto al uso de energía en edificios (Pérez-Lombard, Ortiz, González y Maes, 2009). Dichas certificaciones juegan un papel importante en la mejora de la EE, ya que permiten valorar y comparar diferentes edificios y sus características en lo relativo a demanda y consumo energético (LópezAsiain, García, Fernández y de Tejada Alonso, 2020). Las clasificaciones de energía surgieron también como herramientas para minimizar el consumo de energía y las emisiones de gases de efecto invernadero y promover una mayor transparencia con respecto al uso de energía en los edificios (Reus-Netto, Mercader-Moyano y Czajkowski, 2019).

En Argentina, el primer antecedente de aval legal en la aplicación de normativas relativas a EE, es la Ley Provincial de Buenos Aires $n^{\circ} 13.059 / 03$ (2003) y su Decreto reglamentario 1030/10 (2010). Dicha ley establece la exigencia, para toda construcción nueva tramitada en la provincia, de incluir verificaciones de los niveles de desempeño higrotérmico estipulados por normas IRAM de la serie 11600. De escasa aplicación, los principales problemas de esta ley fueron las variadas exigencias constructivas, la autonomía de las municipalidades y el sistema descentralizado de control e inspección (Chevez, Martini y Discoli, 2016).

En el año 2007, se crea el "Programa Nacional de Uso Racional y Eficiente de la Energía" (PRONUREE), Decreto $140 / 2007$, cuyo objetivo fue propender a un uso eficiente de la energía, lo que implicó una adecuación de los sistemas de producción, transporte, distribución, almacenamiento y consumo de energía, buscando conseguir el mayor desarrollo sostenible con los medios tecnológicos disponibles para aminorar el impacto ambiental y optimizar la conservación de energía, percibiendo así una reducción de costos (Ministerio de Energía y Minería [MINEM], s.f.).

En el año 2009, aparece la primera edición la norma IRAM 11900: "Etiqueta de Eficiencia Energética de calefacción para edificios". La norma establecía ocho niveles de eficiencia térmica de la envolvente siguiendo el formato de las etiquetas de EE de los electrodomésticos. Proponía una metodología simplificada para el cálculo del nivel de EE de las envolventes de los edificios susceptibles de ser calefaccionados (IRAM, 2009).

A posteriori se desarrollan otros antecedentes legales, como la Ley No 3246 (2009): "Consumo de la Energía Reducción y Optimización", del Gobierno de la Ciudad Autónoma de Buenos Aires; la Ordenanza 8757 de la Municipalidad de Rosario (2011): "Aspectos Higrotérmicos y Demanda Energética de las Construcciones"; y la Ley 4458 (2012): "Normas de acondicionamiento térmico en la construcción de edificios", de la Ciudad Autónoma de Buenos Aires.

En 2017 se realizó una nueva versión de la norma IRAM 11900, la cual establece el cálculo de las prestaciones energéticas para inmuebles de uso residencial, integrando mayor cantidad de variables con respecto a su primera versión: calefacción, refrigeración, agua caliente sanitaria, iluminación interior y energías renovables. La principal diferencia con respecto a su anterior edición es que esta contempla estrategias de EE tanto pasivas como activas.

Un año antes, por medio de un Acta Acuerdo firmada entre el Ministerio de Minería y Energía de la Nación (Subsecretaría de Ahorro y Eficiencia Energética), el Gobierno de la Provincia de Santa Fe (Secretaria de Estado de Energía), la Municipalidad de la Ciudad de Rosario y los Colegios Profesionales de la Provincia de Santa Fe (Ingeniería Civil, Ingenieros Especialistas, Arquitectos, y Maestros Mayores de Obra y Técnicos), deciden cooperar en políticas públicas de EE en usos finales. Este documento da lugar, entre otros objetivos, a la realización de una prueba piloto de certificación de EE en inmuebles destinados a vivienda, durante el año 2017 en la ciudad de Rosario (Acta Acuerdo, 2016).

Para implementar la primera prueba piloto, se capacitó a profesionales en un proceso de certificación, para luego evaluar viviendas ya construidas. En agosto de 2018, se efectuó una segunda prueba en la ciudad de Santa Fe. A partir de ambas experiencias y de un convenio celebrado con la Secretaría de Energía de la Nación, el modelo se expandió a otras ciudades del país, con el fin de ajustar el procedimiento de cálculo y el aplicativo informático a partir de las diferentes zonas bioclimáticas y las distintas 
técnicas constructivas. Las pruebas se llevaron a cabo en las ciudades de San Carlos de Bariloche, Mendoza-Godoy Cruz y San Miguel de Tucumán. En 2019 llega también a la ciudad de Salta (Gobierno de Santa Fe, 2020).

Con el propósito de introducir la etiqueta de EE en el país, en el año 2018 surge el Programa Nacional de Etiquetado de Viviendas, basado en la Norma IRAM 11900. Se trata de obtener una estimación del consumo de energía primaria en hogares residenciales ya construidos, para satisfacer las demandas generadas por requerimientos de calefacción, refrigeración, producción de agua caliente sanitaria e iluminación, durante un año típico. De esta manera, los individuos podrán conocer el nivel de EE de una vivienda, volviéndose esta nueva herramienta una pieza muy importante de decisión a la hora de comprar, alquilar o construir un inmueble destinado a vivienda. Cabe destacar que se constituye igualmente como instrumento útil para evaluar un nuevo proyecto o plantear reformas en uno existente.

La Ley 13903 (2019) "Etiquetado de eficiencia energética de inmuebles destinados a vivienda" sancionada por la Legislatura de la Provincia de Santa Fe en noviembre de 2019, es el principal marco legal y antecedente a nivel nacional, que consolida una política específica de EE. Dicha ley reglamenta la aplicación del Índice de Prestaciones Energéticas (IPE) y el Etiquetado Energético de inmuebles destinados a viviendas, crea un Registro de Etiquetas, un Registro de Certificadores y una Comisión con carácter de órgano asesor, e incluso determina bonificaciones para su implementación. A la fecha, aún se espera la adhesión a la Ley de Comunas o Municipios, y tanto la norma IRAM 11900 (2017) como el Programa Nacional de Etiquetado de Viviendas no tienen una implementación de carácter obligatorio en el territorio nacional, constituyéndose sólo como una herramienta para profesionales y usuarios.

El objetivo del presente trabajo es comparar el IPE, definido por el Programa Nacional de Etiquetado de Viviendas, con las Estrategias Pasivas de Diseño Arquitectónico (EPDA), establecidas en la primera modificación a la norma IRAM 11900 (2019), para evaluar la incidencia de dichas estrategias en el valor del IPE de una vivienda.

\section{METODOLOGÍA}

La metodología empleada en la investigación aquí expuesta corresponde a un análisis normativo y al estudio de un caso. En una primera instancia, se realiza un análisis normativo completo de IRAM 11900:2017 y de su modificación llevada a cabo en 2019. Se estudia la etiqueta de EE y la ponderación EPDA (estas últimas planteadas en la primera modificación). Luego, se analiza el aplicativo web desarrollado por la Secretaría de Energía para la obtención de una etiqueta de EE que determina la "Clase de Eficiencia Energética", lo cual da como resultado

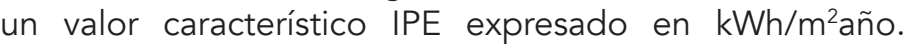

Se examina la incidencia de las estrategias de diseño pasivo y activo en el valor IPE y se procede a comparar las variables pasivas que inciden en EPDA y en IPE. Por último, se analizan ambas variables (IPE y EPDA) a partir de un caso de estudio. De ello, se obtiene el valor IPE y, por ende, la Clase de EE a través del aplicativo web. Por otra parte, se efectúa la ponderación EPDA mediante el calculador EBioDA (Fernández y Garzón, 2019) para una mejor comprensión de los resultados. A continuación, se desarrolla una propuesta de rehabilitación energética a partir de estrategias pasivas y se calculan nuevamente, por medio de la misma metodología, los valores recién citados. Para terminar, se procede a comparar la mejora porcentual de IPE y EPDA a partir de las mejoras realizadas.

\section{ANÁLISIS DE LOS MECANISMOS DE EVALUACIÓN DE EE EN VIVIENDAS}

\section{SOBRE LA NORMA IRAM 11900:2017}

En diciembre de 2017, fue publicada la nueva edición de la norma IRAM 11900 "Prestaciones energéticas en viviendas. Método de cálculo". Este documento, elaborado por especialistas y sectores profesionales que forman parte del Subcomité de eficiencia energética en edificios, establece un cambio de paradigma en lo que respecta a la evaluación de la EE (IRAM, 2017). En ella se fijan las bases para el etiquetado en viviendas. En enero de 2019, se introdujo una modificación en la norma, la cual quedó definida como: "Prestaciones energéticas en viviendas. Método de cálculo y etiquetado de eficiencia energética".

Allí se precisa un método de cálculo para determinar la prestación energética de unidades residenciales, donde la noción de "prestaciones energéticas" se refiere al uso final de energía convencional que contribuye a la demanda energética de la vivienda mediante los siguientes servicios: calefacción, refrigeración, iluminación artificial interior y calentamiento de agua sanitaria. Se mencionan las estrategias bioambientales de diseño como una manera efectiva de contribuir a la EE, encontrándose expuestas en primer lugar al momento de enunciar el cálculo de las prestaciones energéticas, de la siguiente manera:

Los recursos pasivos de diseño, incorporados en el proyecto arquitectónico y la composición de elementos constructivos, contribuyen de manera efectiva a la eficiencia energética de las viviendas y a la reducción de la demanda de energía a través de distintos mecanismos, tales como: la disminución de la demanda de energía requerida para calefacción, a través de: la conservación de calor en épocas de bajas temperaturas con aislación térmica, la forma y el diseño compacto y el control de la renovación de aire; la captación de la radiación solar en épocas cuando su contribución a la calefacción es favorable; el control del ingreso de radiación solar en el período estival mediante, la aislación térmica, los techos de color claro y la protección solar en superficies vidriadas; la incorporación de refrescamiento natural con ventilación cruzada y selectiva. En este contexto, las estrategias bioambientales y los recursos pasivos de diseño, conjuntamente con los 
aportes de las instalaciones eficientes y la contribución adicional de energías renovables integradas al proyecto, contribuyen a mejorar la eficiencia energética de la vivienda (IRAM, 2017, p.11).

Se puede observar que IRAM considera primordial la contemplación de las estrategias pasivas de diseño.

Inmediatamente después de las estrategias bioambientales, se menciona el requerimiento específico de energía primaria de climatización (calefacción y refrigeración), el que comprende el cálculo para la evaluación térmica y evaluación de la EE para climatización. En tercer lugar, se encuentra el cálculo de agua caliente sanitaria cuyo objetivo es caracterizar los sistemas empleados y determinar su eficiencia; en cuarto, la iluminación artificial interior; y, por último, el aporte por el uso de energías renovables, donde solo se contempla la energía solar: solar térmica, para el calentamiento de agua, y fotovoltaica, para la producción de electricidad.

A partir del resultado de los cálculos antes mencionados quedan definidas las prestaciones energéticas totales de la vivienda, como la diferencia entre el requerimiento específico de energía primaria (demanda anual de energía para climatización, calentamiento de agua e iluminación) y las contribuciones de energía generada por fuentes renovables.

\section{ETIQUETA DE EFICIENCIA ENERGÉTICA DE IRAM 11900}

La etiqueta de EE se obtiene como resultado del cálculo de las prestaciones energéticas (PE) de la vivienda. Las variables que se consideran en la etiqueta son: requerimiento específico anual de energía primaria para climatización $\left(E P_{C}\right)$, requerimiento específico anual de energía primaria para ACS $\left(E_{\text {A.C.S. }}\right)$, requerimiento específico anual de energía primaria para iluminación $\left(E_{\|}\right)$, contribución de energía renovable $\left(E P_{R E N}\right)$ y valoración de nivel de Estrategias Pasivas de Diseño Arquitectónico (EPDA).

La clase de EE queda categorizada de la "A" a la "G". Esta se determina a partir del valor numérico $\mathrm{PE}$ en $\mathrm{KWh} / \mathrm{m}^{2}$, el cual contempla en su cálculo: contribución de energías renovables, en Kwh; requerimiento de energía primaria para la climatización, en Kwh; requerimiento de energía primaria para la producción de ACS, en Kwh; y requerimiento de energía primaria para iluminación interior, en Kwh.

Si bien la norma prioriza la ponderación de estrategias pasivas EPDA, éstas no inciden en el resultado de la etiqueta. Cabe destacar que en la norma se mencionan las estrategias pasivas en primer lugar, y en el diseño de la etiqueta quedan relegadas al final.

\section{ESTRATEGIAS PASIVAS DE DISEÑO ARQUITECTÓNICO (EPDA) DE IRAM 11900}

Se definen a las EPDA como: "Características del diseño arquitectónicoy de los elementos constructivos que adecuan

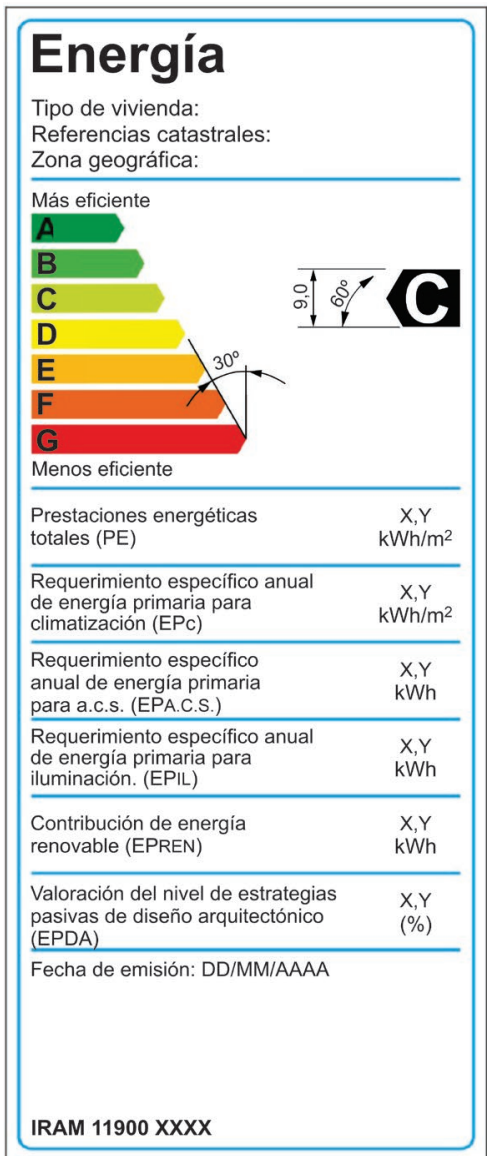

Figura 1. Modelo de Etiqueta de EE.

Fuente: Modificación n 1 a la norma IRAM 11900: 2017

el edificio a las condiciones climáticas y ambientales, y permiten mejorar la sensación de confort higrotérmico y reducir la demanda de energía convencional" (IRAM, 2019, p. 2). Las EPDA posibles de evaluar son: aislación térmica; inercia térmica; protección solar; ventilación natural; captación solar; humidificación; y entorno. La ponderación de la relevancia de las estrategias bioclimáticas depende del clima de la región donde se emplaza el edificio.

Para determinar la ponderación de las EPDA, se identifican una serie de parámetros característicos, denominados recursos arquitectónico-constructivos (RAC), que reducen la demanda de energía y optimizan el confort higrotérmico mediante el acondicionamiento natural del edificio en los períodos de verano e invierno (IRAM, 2019). La relevancia de cada RAC varía en función de la caracterización climática de la zona analizada. Por ejemplo, para el recurso "Aislación térmica del piso" en un clima cálido, el puntaje es uno (1) para cualquier tipo de solución constructiva, mientras que para un clima frío, este puntaje varía desde cero (0), piso sin aislación, hasta uno (1), piso con aislación térmica total. Los RAC considerados son: aislación térmica del techo, aislación térmica de muros exteriores, aislación térmica de ventanas, aislación térmica del piso, inercia térmica del techo, inercia térmica de muros exteriores, inercia térmica del piso, color externo del techo, color externo de muros, 
sombreado de ventanas, sistemas solares pasivos directos, sistemas solares pasivos indirectos, altura piso-techo, contacto con otras viviendas, obstáculos exteriores, sol verano, forma compacta, proporción de ventana en muros, ventilación cruzada, ventilación selectiva, suelo exterior vegetación, obstáculos contra viento, superficie ventana en techo y nivel de hermeticidad.

El equipo de trabajo GHabSS (Grupo Hábitat Sustentable y Saludable) de la FAU-UNT desarrolló un calculador C-EBioDA (Fernández y Garzón, 2019), para simplificar y sistematizar la obtención de la ponderación de estrategias pasivas para la ciudad de San Miguel de Tucumán. Este lleva a cabo una sencilla sistematización, a través de una planilla de cálculo, de todas las variables cualitativas con sus correspondientes valores que inciden en la ponderación EPDA. Para su ejecución, las únicas variables a seleccionar son las calidades de los elementos constructivos para cada RAC. Gracias a este proceso se obtiene como resultado el puntaje de dicho recurso. Las ventajas de su uso son la simplificación del proceso de cálculo y la reducción de posibles errores por la cantidad de valores numéricos involucrados. Empleando dicho calculador, también es posible calcular, a partir del porcentaje total de EPDA, la incidencia porcentual de cada estrategia pasiva; de manera que se consigue una visualización rápida que permite definir estrategias de mejora.

\section{SOBRE EL APLICATIVO INFORMÁTICO NACIONAL}

El aplicativo informático nacional es una herramienta on line, basada en la norma IRAM 11900, diseñada para realizar la evaluación de EE en una vivienda en cualquier lugar del país a partir de un relevamiento de esta y obtener la Etiqueta correspondiente, conforme a los lineamientos establecidos a nivel nacional. Asimismo, contribuye a formular recomendaciones de mejora y cuantificar el impacto de estas en términos de potenciales ahorros (Ministerio de Desarrollo Productivo, s.f.). Este aplicativo clasifica la EE a la vivienda en siete categorías.

Para validar el aplicativo y llevar a cabo ajustes que garanticen su correcta adaptación a todo el territorio nacional, contemplando las particularidades climáticas, socioeconómicas y de las prácticas constructivas, se efectuaron pruebas en localidades de diferentes provincias y regiones del país. Se ejecutó a modo de prueba piloto la certificación de 1410 viviendas (Ministerio de Desarrollo Productivo, s.f.), a fin de ponderar los valores actuales y, a partir de ello, definir los distintos rangos de la etiqueta. Sin embargo, el aplicativo aún no está disponible para todas las localidades del país.

\section{ÍNDICE DE PRESTACIONES ENERGÉTICAS (IPE)}

El IPE es un valor característico del inmueble, expresado en $\mathrm{kWh} / \mathrm{m}^{2}$ año. Representa el requerimiento teórico de energía primaria para satisfacer las necesidades de calefacción en invierno, refrigeración en verano, calentamiento de agua sanitaria e iluminación, durante un año y por metro cuadrado de superficie, bajo condiciones normalizadas de uso (Ministerio de Desarrollo Productivo, s.f.).

En el valor IPE inciden distintas variables de diseño. Ellas son: orientaciones, materialización de la envolvente, características de las aberturas, terminación de superficies exteriores e interiores, altura piso-techo, elementos de sombreado, artefactos de iluminación y tipo de accionamiento, características de los equipos de climatización, sistema de calentamiento de agua, uso de energía fotovoltaica y calentamiento de agua con energía solar.

Estas variables, pensadas como estrategias para el ahorro energético, pueden clasificarse en activas y pasivas. Las pasivas son aquellas que deben implementarse en primera instancia para reducir las demandas energéticas (Martín-Consuegra, Oteiza, Alonso, Cuerdo-Vilches y Frutos, 2014). Existen estudios que demuestran que la aplicación de estrategias pasivas disminuyen considerablemente el consumo de energía de un edificio construido (Aguilera, Viñas, Rodríguez y Varela, 2018). Es importante destacar que dicha reducción es independiente de la fuente de energía empleada.

En la Tabla 1, se puede observar la clasificación de las variables incidentes en el valor característico IPE.

\begin{tabular}{|c|c|c|}
\hline Variables incidentes & $\begin{array}{l}\text { Estrategia } \\
\text { Pasiva }\end{array}$ & $\begin{array}{l}\text { Estrategia } \\
\text { Activa }\end{array}$ \\
\hline Composición de la envolvente & $\bullet$ & \\
\hline Características de las aberturas & $\bullet$ & \\
\hline Orientaciones & $\bullet$ & \\
\hline $\begin{array}{c}\text { Terminación de superficies } \\
\text { exteriores }\end{array}$ & • & \\
\hline $\begin{array}{c}\text { Terminación de superficies } \\
\text { interiores }\end{array}$ & $\bullet$ & \\
\hline Altura piso- techo & $\bullet$ & \\
\hline Protecciones solares & $\bullet$ & \\
\hline Artefactos de iluminación & & $\bullet$ \\
\hline $\begin{array}{l}\text { Tipo de accionamiento de } \\
\text { artefactos de iluminación }\end{array}$ & & $\bullet$ \\
\hline $\begin{array}{c}\text { Características de los equipos de } \\
\text { refrigeración }\end{array}$ & & $\bullet$ \\
\hline $\begin{array}{c}\text { Características de los equipos de } \\
\text { calefacción }\end{array}$ & & $\bullet$ \\
\hline Sistema de ACS & & $\bullet$ \\
\hline Uso de energía fotovoltaica & & $\bullet$ \\
\hline $\begin{array}{c}\text { Calentamiento de agua con } \\
\text { energía solar }\end{array}$ & & $\bullet$ \\
\hline
\end{tabular}

Tabla 1. Clasificación de las variables incidentes en IPE.

Fuente: Elaboración de los autores. 


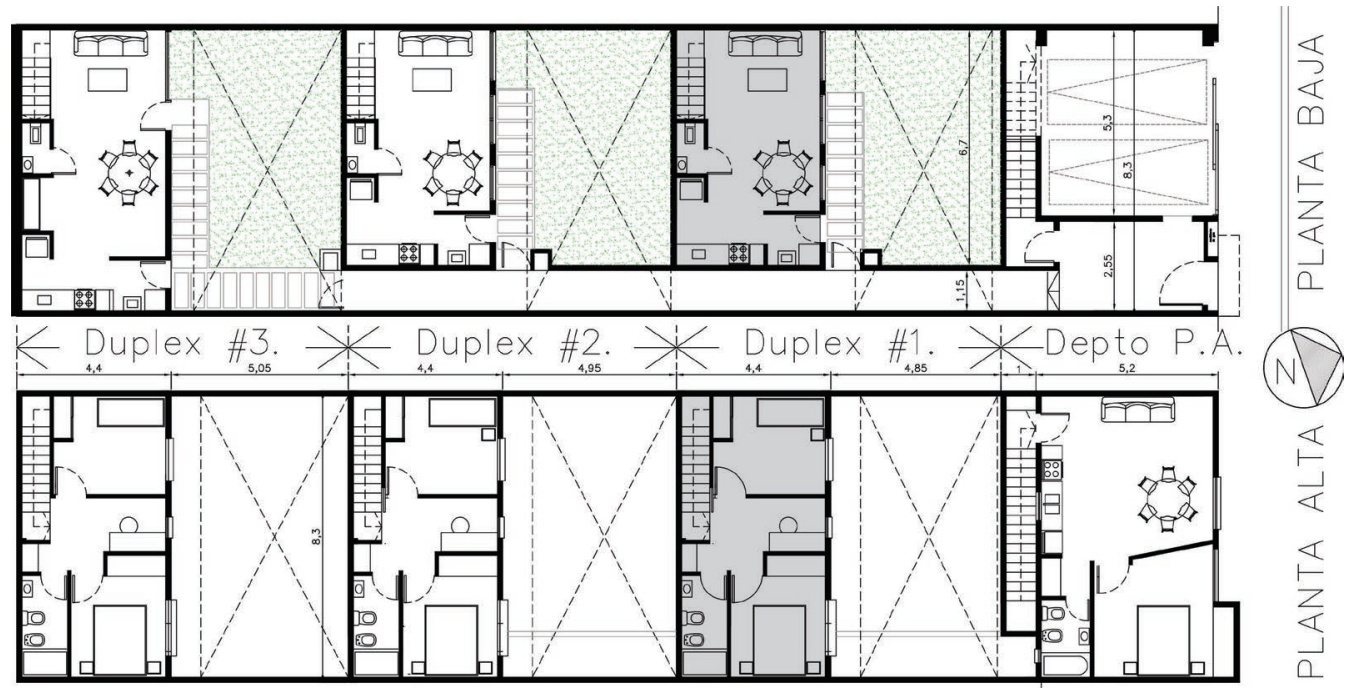

Figura 2. Planta baja y alta del PH.

Fuente: Elsinger y Garzón (2019, p. 250)

\section{COMPARACIÓN DE VARIABLES DE DISEÑO PASIVO EN IPE Y EPDA}

Tanto para el valor característico IPE como para la valoración EPDA inciden diversas variables de diseño pasivo, ya que en EPDA no se consideran las activas. A continuación (Tabla 2), se enumeran todas estas variables y se indica si están contempladas en cada valor.

De acuerdo a la Tabla 2, se advierte que existen variables pasivas no contempladas en el IPE, como también que la variable "Color de las superficies interiores", en cuanto estrategia pasiva para el ahorro de energía de iluminación, está contemplada en el IPE y no en EPDA.

\section{ANÁLISIS Y VALORACIÓN DE LAS VARIABLES PASIVAS A PARTIR DE UN CASO DE ESTUDIO}

Para analizar la correlación de ambas variables (IPE y EPDA), se toma un caso de estudio como ejemplo de aplicación. El caso se ubica en la Ciudad de San Miguel de Tucumán, provincia de Tucumán, al noroeste del país. Se localiza específicamente en la zona bioclimática $\mathrm{llb}$, donde el verano es la estación crítica, con temperaturas medias superiores a los $24^{\circ} \mathrm{C}$, máximas por encima de los $30^{\circ} \mathrm{C}$ y amplitudes menores a $14^{\circ} \mathrm{C}$. El invierno es más seco y presenta bajas amplitudes y temperaturas medias comprendidas entre $8^{\circ} \mathrm{C}$ y $12^{\circ} \mathrm{C}$ (IRAM, 2012). El estudio se aplica a una vivienda unifamiliar, cuyas prestaciones energéticas habían sido analizadas previamente para generar una propuesta de rehabilitación térmicoenergética, que fue publicada en ASADES 2019 por el Arq. David Elsinger y la Dra. Beatriz Garzón.

\section{DESCRIPCIÓN DEL CASO}

La vivienda forma parte de una propiedad horizontal. La propiedad está constituida por tres unidades tipo "dúplex" desarrolladas en dos niveles y una unidad en planta

\begin{tabular}{|c|c|c|}
\hline Variable & EPDA & IPE \\
\hline $\begin{array}{c}\text { Aislación térmica de } \\
\text { la envolvente }\end{array}$ & Contemplada & Contemplada \\
\hline $\begin{array}{c}\text { Inercia térmica de la } \\
\text { envolvente }\end{array}$ & Contemplada & Contemplada \\
\hline $\begin{array}{c}\text { Color de las } \\
\text { superficies } \\
\text { exteriores }\end{array}$ & Contemplada & Contemplada \\
\hline $\begin{array}{c}\text { Color de las } \\
\text { superficies interiores }\end{array}$ & No contemplada & Contemplada \\
\hline Protecciones solares & Contemplada & Contemplada \\
\hline Ventilación natural & Contemplada & No contemplada \\
\hline Humidificación & Contemplada & No contemplada \\
\hline Captación solar & Contemplada & No contemplada \\
\hline Entorno & Contemplada & No contemplada \\
\hline
\end{tabular}

Tabla 2. Estrategias pasivas presentes en IPE y/o en EPDA. Fuente: Elaboración de los autores.

alta (Figura 2). Además, posee un acceso común, una servidumbre de paso para llegar a todas las unidades y un garaje para dos vehículos. Se ubica en disposición nortesur, con la totalidad de sus aberturas dispuestas hacia el norte (Figura 3).

La construcción existente fue realizada luego de la demolición de una vivienda, por lo tanto, se reciclaron las medianeras (Este y Oeste) existentes resueltas en mampostería de $30 \mathrm{~cm}$ de espesor de ladrillo macizo común con al menos una cara revocada (cara interior). El resto de la envolvente vertical se resolvió en mampostería de $20 \mathrm{~cm}$ de ladrillos cerámicos huecos $(18 \times 18 \times 33 \mathrm{~cm})$ con ambas 

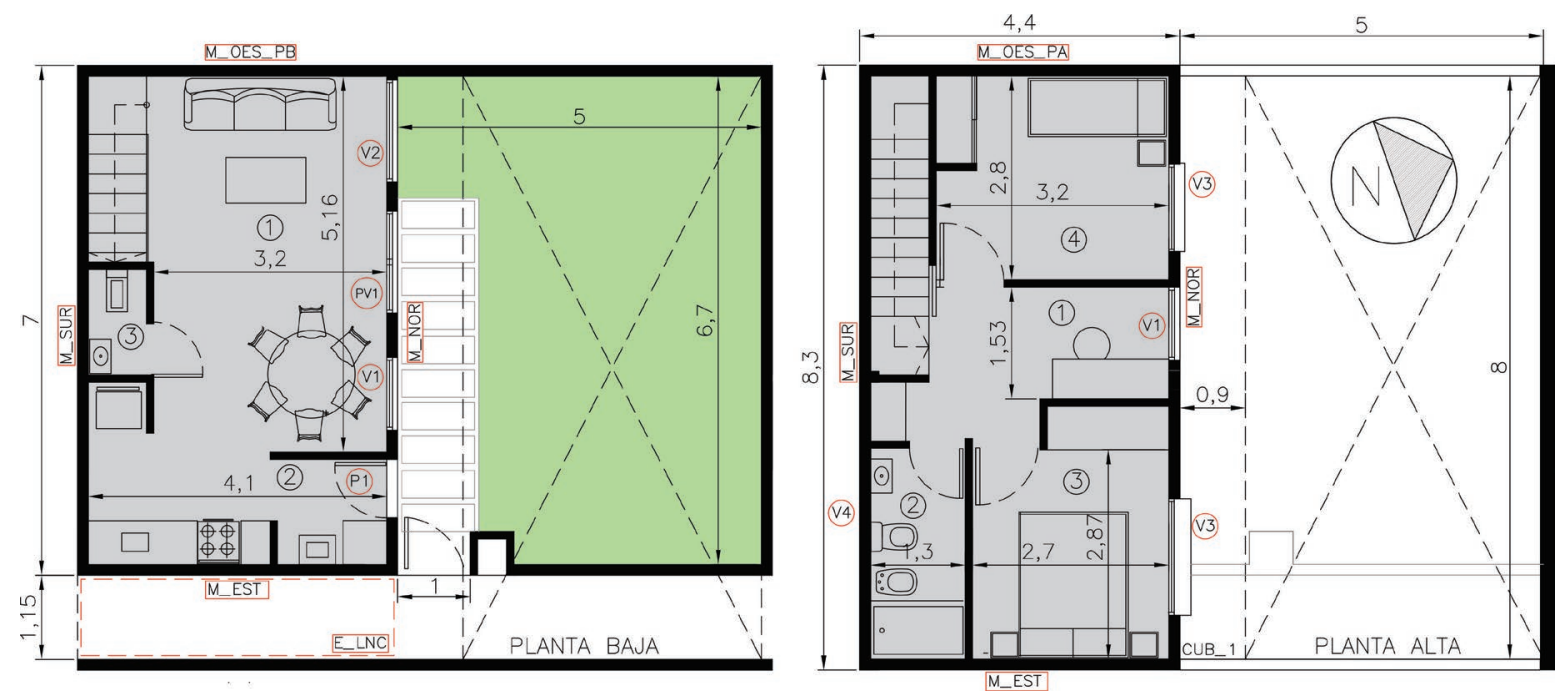

Figura 3. Planta baja y alta de la unidad de estudio.

Fuente: Elsinger y Garzón (2019, p. 251)

caras revocadas. La edificación posee una estructura de hormigón armado independiente y una cubierta de chapa sinusoidal calibre 24, con estructura de perfiles metálicos " $\mathrm{C}$ " y $5 \mathrm{~cm}$ de lana de vidrio colocada en contacto con las chapas. La cubierta se conforma en un único faldón con pendiente hacia el norte, que posee un voladizo de $0.90 \mathrm{~m}$ hacia el patio interno de cada una de las propiedades. Las divisiones interiores están resueltas con diversas técnicas: mampostería de 15 $\mathrm{cm}$ de ladrillo común en planta baja, mampostería de $8 \mathrm{~cm}$ de bloques huecos y también tabiques de placa de yeso cartón con estructura de perfiles metálicos de chapa galvanizada de $10 \mathrm{~cm}$ de espesor en planta alta. Ambas plantas poseen pisos y zócalos cerámicos. Las superficies interiores tienen revoque fino más pinturas en colores claros y los cielorrasos son de yeso aplicados sobre losa en planta baja y suspendido sobre perfiles de chapa galvanizada y cierres de placas roca-yeso en planta alta. Las superficies verticales exteriores poseen un revoque grueso con terminación "salpicado", que posee color propio, en este caso símil "beige claro" (Elsinger y Garzón, 2019).

\section{PROPUESTA DE REHABILITACIÓN}

La propuesta de rehabilitación contempla las siguientes medidas:

- Incremento en la aislación térmica de la envolvente (Tabla 3).

- Reducción de superficie vidriada para disminuir la captación solar. Para esto, se anula una de las ventanas de planta baja orientada hacia el norte (Figura 3: V1Planta Baja).

- Generación de ventilación cruzada. Esto se logra a partir de la apertura de dos ventiluces en la cara sur del edificio: uno en planta baja y otro en primer piso.

\begin{tabular}{|c|c|c|}
\hline $\begin{array}{l}\text { Elemento de la } \\
\text { envolvente }\end{array}$ & Situación original & $\begin{array}{l}\text { Propuesta de } \\
\text { rehabilitación }\end{array}$ \\
\hline Carpinterías & $\begin{array}{l}\text { Corredizas con vidrio } \\
\text { incoloro } 3 \mathrm{~mm}\end{array}$ & De abrir con DVH \\
\hline M_Nor & \multirow{3}{*}{$\begin{array}{l}\text { Ladrillo cerámico } \\
\text { hueco revocado en } \\
\text { ambas caras }(20 \mathrm{~cm})\end{array}$} & \multirow{3}{*}{$\begin{array}{l}\text { Incorporación de } \\
\text { aislante en la cara } \\
\text { exterior: } 7 \mathrm{~cm} \\
\text { de poliestireno } \\
\text { expandido + placa } \\
\text { cementicia }\end{array}$} \\
\hline M_Sur & & \\
\hline M_Est_PB & & \\
\hline M_Est_PA & \multirow[b]{2}{*}{$\begin{array}{l}\text { Ladrillo cerámico } \\
\text { macizo revocado en } \\
\text { la cara interior (30 } \\
\mathrm{cm} \text { ) }\end{array}$} & \multirow{2}{*}{$\begin{array}{c}\text { Incorporación de } \\
\text { aislante en la cara } \\
\text { interior (por ser } \\
\text { medianeros): } 5 \mathrm{~cm} \\
\text { de lana de vidrio }+ \\
\text { placa de roca-yeso } \\
\text { de } 12 \mathrm{~mm}\end{array}$} \\
\hline M_Oest & & \\
\hline Techo & $\begin{array}{c}\text { Chapa galvanizada } \\
\text { sinusoidal }+5 \mathrm{~cm} \\
\text { de lana de vidrio }+ \\
\text { cielorraso de roca- } \\
\text { yeso } 8 \mathrm{~mm}\end{array}$ & $\begin{array}{c}\text { Incorporación de } \\
5 \mathrm{~cm} \text { más de lana } \\
\text { de vidrio (esta } \\
\text { se coloca desde } \\
\text { la cara exterior } \\
\text { desmontando } \\
\text { las chapas de la } \\
\text { cubierta) }\end{array}$ \\
\hline E_LNC & $\begin{array}{l}\text { Losa de viguetas } \\
\text { pretensadas } \\
\text { con bovedillas } \\
\text { de poliestireno } \\
\text { expandido }(17 \mathrm{~cm})\end{array}$ & $\begin{array}{c}\text { Incorporación en } \\
\text { la cara inferior de } \\
5 \mathrm{~cm} \text { de lana de } \\
\text { vidrio + placa de } \\
\text { roca-yeso }\end{array}$ \\
\hline
\end{tabular}

Tabla 3. propuestas de aislación térmica en la envolvente.

Fuente: Elaboración de los autores. 

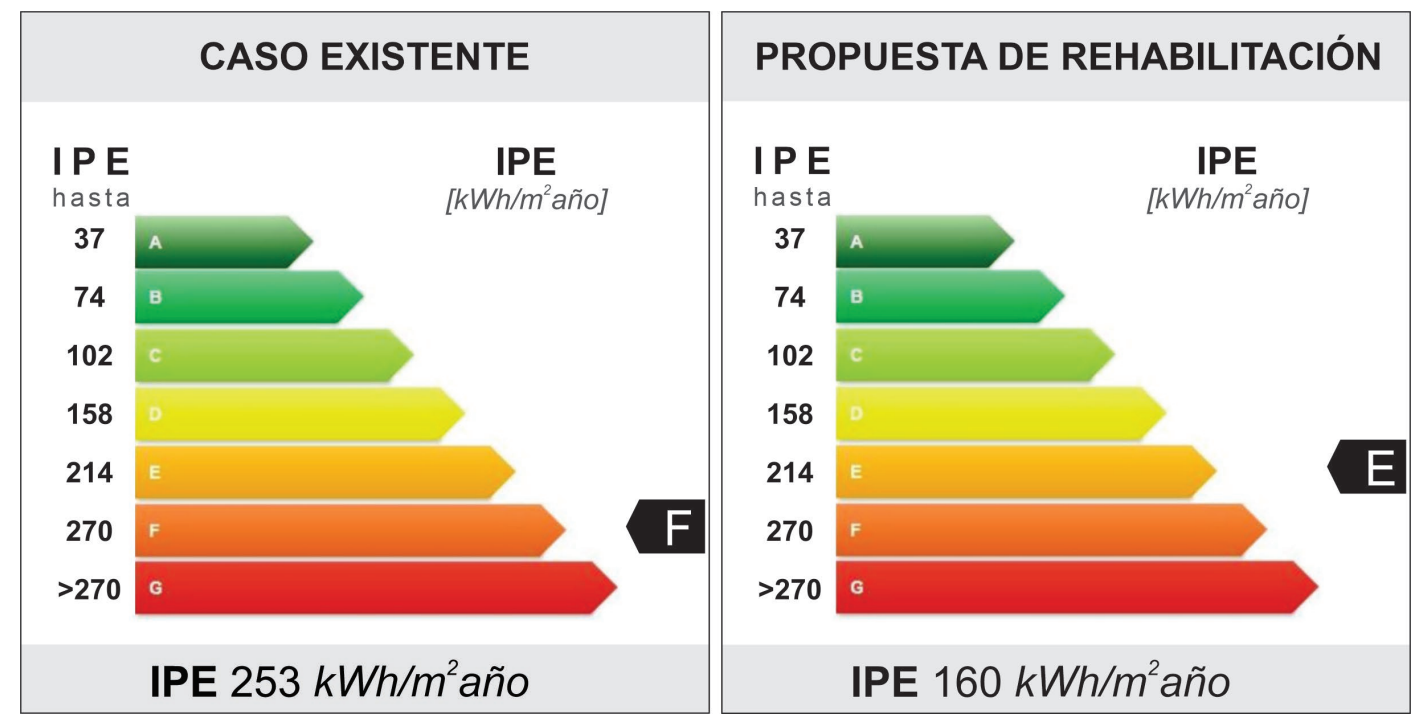

Figura 4. Valor IPE para caso existente y propuesta de rehabilitación. Fuente: Elaboración de los autores

\begin{tabular}{|lr|lr|}
\hline \% EPDA & & & \\
\hline & $\mathbf{2 3 . 7 6} \%$ & $\%$ EPDA & $\mathbf{4 8 . 6 9 \%}$ \\
\hline Aislación térmica & & & $83.75 \%$ \\
Inercia térmica & $28.75 \%$ & Aislación térmica & $24.90 \%$ \\
Protección solar & $24.90 \%$ & Inercia térmica & $30.70 \%$ \\
Ventilación natural & $30.70 \%$ & Protección solar & $32.50 \%$ \\
Captación solar & $5.00 \%$ & Ventilación natural & $32.20 \%$ \\
Humidificación & $17.20 \%$ & Captación solar & $0.00 \%$ \\
Entorno & $0.00 \%$ & Humidificación & $35.00 \%$ \\
\hline
\end{tabular}

Figura 5. Determinación de \% EPDA a partir del calculador EBioDA.

Fuente: Elaboración de los autores

\section{COMPARACIÓN DE RESULTADOS IPE Y VALORACIÓN EPDA PARA AMBOS CASOS}

Se calcula el valor IPE a través del aplicativo web. Los resultados que se obtienen son los siguientes: para la

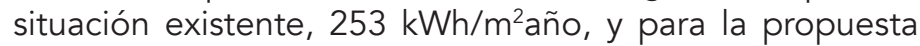

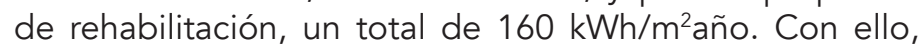
el prototipo original queda en la clasificación " $F$ " y la propuesta de rehabilitación, en la "E".

Posteriormente, se realiza la valoración EPDA en ambos casos, empleando como herramienta el calculador C-EBioDA (Fernández y Garzón, 2019). Los valores porcentuales obtenidos son de $23,76 \%$, para el caso original, y 48,69\%, para la propuesta de rehabilitación.

Se puede apreciar (Figura 5) que las mejoras sustanciales se dan en la aislación térmica (de $28,75 \%$ a $83,75 \%$ ), en la ventilación natural (de $5 \%$ a $32 \%$ ) y en la captación solar (de $17 \%$ a $32 \%$ ). Esto se debe a las estrategias de rehabilitación implementadas. En aislación, se optimiza el comportamiento de la envolvente en su totalidad (muros, techo y ventanas). La ventilación natural se logra incorporando ventiluces en el muro sur, lo cual favorece la ventilación cruzada en casi toda la vivienda. Por último, se disminuye la captación solar, al reducir la superficie vidriada, eliminando una ventana orientada hacia el norte.

Al comparar ambos valores, se observa que, en cuanto a la valoración de estrategias pasivas, el caso analizado mejora en un $52,1 \%$, mientras que, en términos del IPE, solo lo hace en un $36,6 \%$. En consecuencia, las optimizaciones en las estrategias pasivas no implican una mejora -en el mismo grado-, en las prestaciones energéticas de la vivienda.

\section{DISCUSIÓN}

Es fundamental considerar que cualquier intervención en una vivienda genera incrementos en el confort térmico, lo 
que suscita un amplio margen de actuación (Fernández, Rubio y Guevara, 2019). La rehabilitación energética de viviendas está orientada a mejorar las condiciones edilicias de acuerdo con las posibilidades económicas del usuario, las características del edificio y otras posibles limitaciones (Pérez Fargallo, Calama Rodríguez y Flores Alés, 2016). El compromiso de invertir en la rehabilitación de viviendas como una mejora de la EE del sector residencial, no debe recaer en las buenas intenciones del usuario sino que debe estar acompañado por políticas de ayudas públicas reales y de cuantía proporcional al coste exigido para que el proceso de modernización térmica-energética de edificios sea sostenible y no se transforme en un problema social (Lizundia, Etxepare, Sagarna y Uranga, 2018)

La propuesta de rehabilitación energética aquí expuesta es factible desde el punto de vista tecnológico y socioeconómico local. Se encuentran, no obstante, limitaciones diversas, entre ellas: la disposición del edificio en el terreno (entre medianeras); la falta de materiales y/o productos específicos del mercado local (aislantes térmicos, carpinterías de altas prestaciones, etc.); y limitaciones tecnológicas, propias de los conocimientos de la práctica constructiva local.

Por otro lado, las conclusiones preliminares obtenidas apuntan a reforzar la baja incidencia de las estrategias pasivas en la etiqueta de EE edilicia. Dado que, en este caso, sólo se han analizado las variaciones producidas por dichas estrategias, sería relevante analizar también la incidencia las estrategias activas en la etiqueta energética con el fin de llevar a cabo una ponderación más adecuada.

\section{CONCLUSIONES}

A partir del análisis de la norma IRAM 11900:2017, como así también del aplicativo web para el etiquetado energético, que permitió evaluar principalmente la ponderación de las estrategias pasivas de diseño arquitectónico, y tras el posterior análisis de un caso de estudio y su consiguiente propuesta de rehabilitación energética basada en estrategias pasivas, se verifica que, si bien este tipo de intervenciones tienen incidencia, no revisten primordial importancia.

El Programa de Etiquetado de Viviendas constituye, sin duda alguna, una auténtica política nacional para hacer frente a las condiciones energéticas del mundo contemporáneo. En camino a una implementación del Programa de Etiquetado de Viviendas en toda la Argentina (Subsecretaría de Energías Renovables y Eficiencia Energética, s.f.), se hace necesario revisar las formas en que se ponderan los datos que sirven para obtener el IPE, para obtener un etiquetador que se adecue a las diferentes realidades socioeconómicas del país.

El IPE determina la etiqueta de EE de unidades residenciales en Argentina. Aun cuando su implementación no es de carácter obligatorio, se espera que sea de interés colectivo, ya que, al estar reconocida por el mercado inmobiliario, se constituye como un valor agregado para la compraventa, alquiler o construcción de un inmueble destinado a vivienda, en relación con su grado de eficiencia.

Por otra parte, resulta valorable considerar que la mejora de la aislación térmica de la envolvente implica mejoras en el valor IPE, pero esta es la única medida pasiva que conduce a dicho objetivo, dejando de lado otras variables. Sería muy positivo, entonces, que la consideración de las estrategias pasivas tuviera un rol más significativo, a modo de generar conciencia y un mayor conocimiento acerca del bioclimatismo.

Es sumamente cuestionable el hecho de que se contemplen como estrategias de mejoras aquellas que implican costos energéticos de producción y generan residuos importantes (como el cambio de los equipos de climatización o luminarias). La posibilidad de alcanzar los más altos estándares, fundamentales para garantizar un hábitat sano y energéticamente eficiente, es factible a través del diseño pasivo que asegura notables mejoras en el confort natural y en la reducción del uso de energía convencional. Por lo tanto, se requiere el uso de una herramienta que pondere favorablemente las estrategias pasivas y no conlleve a la incorporación de equipos de climatización, equipos de generación de energías renovables $u$ otros sistemas activos, solo para alcanzar una mejor etiqueta.

Por lo expresado, y entendiendo la Etiqueta Energética como una herramienta para plantear procesos de reformas de viviendas, el estudio realizado termina planteándose las siguientes interrogantes: ¿Cuáles son las incidencias finales de las estrategias pasivas y activas en la Etiqueta Energética? ¿Es necesario revisar dichas incidencias a fin de adaptar la Etiqueta a la diversidad de contextos socioeconómicostecnológicos locales de la República Argentina?

\section{AGRADECIMIENTOS}

En primer lugar, se agradece al CONICET (Concejo Nacional de Investigaciones Científicas y Tecnológicas de Argentina) y a la FAU-UNT (Facultad de Arquitectura y Urbanismo - Universidad Nacional de Tucumán), por el fomento a investigadores vinculados a este trabajo. En segundo lugar, se agradece al Dr. Ernesto Kuchen por compartir el conocimiento y fomentar el pensamiento crítico y reflexivo, que dio lugar a esta investigación.

\section{REFERENCIAS BIBLIOGRÁFICAS}

Acta Acuerdo de 2016. (2016). Cooperación en Políticas Públicas de Eficiencia Energética en usos finales. Ministerio de Minería y Energía de la Nación, Gobierno de la Provincia de Santa Fe, Municipalidad de la Ciudad de Rosario y Colegios Profesionales 
de la Provincia de Santa Fe. 27 de octubre de 2016. Recuperado de https://www.santafe.gob.ar/ms/eficienciaenergetica/ wp-content/uploads/sites/25/2018/12/2016-10-27_ACTAACUERDO-MINEM-SEE-MR-COLEGIOS.pdf

Aguilera, P., Viñas, C., Rodríguez, A. y Varela, S. (2018). Análisis de la influencia, en la demanda de climatización, de estrategias pasivas en viviendas con grandes superficies acristaladas, mediante un código de simulación. La casa Farnsworth. Anales de Edificación, 4(3), 34-43. http://dx.doi.org/10.20868/ ade.2018.3798

Alonso-Frank, A. y Kuchen, E. (2017). Validación de la herramienta metodológica de Alonso-Frank \& Kuchen para determinar el indicador de nivel de eficiencia energética del usuario de un edificio residencial en altura, en San Juan - Argentina. Revista Hábitat Sustentable. 7(1), 6-13. Recuperado de http://revistas. ubiobio.cl/index.php/RHS/article/view/2740

Aragón, C. S., De Olivera Pamplona, E., y Medina, J. R. V. (2012). La eficiencia energética como herramienta de gestión de costos: una aplicación para la identificación de inversiones de en eficiencia energética, su evaluación económica y de riesgo. Revista Digital del Instituto Internacional de Costos, (1), 48-73. Recuperado de http://www.revistaiic.org/articulos/numesp/articulo3_esp.pdf

Chevez, P., Martini, I. y Discoli, C. (2016). Avances en la construcción de escenarios energéticos urbanos del sector residencial a partir del análisis detallado de medidas de eficiencia energética de la República Argentina. Congresso Brasileiro de Planejamento Energético XCBPE. Gramado - RS. Recuperado de http://sedici.unlp.edu.ar/bitstream/handle/10915/55713/ Documento_completo.pdf-PDFA.pdf?sequence=3\&isAllowed=y

Decreto 140/2007 (2007). Programa Nacional de Uso Racional y Eficiente de la Energía PRONUREE. Poder Ejecutivo Nacional (P.E.N.). Buenos Aires, Argentina. 24 de diciembre 2007. Recuperado de http://servicios.infoleg.gob.ar/infoleglnternet/ anexos/135000-139999/136078/norma.htm

Elsinger, D. y Garzón B. (2019). Incidencia del rediseño de envolvente en la Etiqueta de Eficiencia Energética de una vivienda existente. XLII Reunión de Trabajo de la Asociación Argentina de Energía Solar, San Salvador de Jujuy, Argentina, 11 al 14 de noviembre. Recuperado de http: //www.exporenovables.com. ar/2019/descargas/actas/tema1/2528.pdf

Fernández, A. y Garzón, B. (2019). C-EBioDA: Calculador de Estrategias Bioclimáticas de Diseño Arquitectónico, según NORMA IRAM 11900. Obra inédita no musical inscripta en Dirección Nacional de Derechos del Autor (DNDA), expediente: EX-2019-89171618- -APN-DNDA\#MJ. Ciudad Autónoma de Buenos Aires (CABA), octubre de 2019.

Fernández, P. X., Rubio, C. y Guevara, F. J. (2019). Rehabilitación energética de viviendas en España: confort térmico y efectividad. Anales de Edificación, 5(1), 37-50. http://dx.doi. org/10.20868/ade.2019.3913

Gobierno de Santa Fe (s.f.). Etiquetado de viviendas. Pruebas piloto. Recuperado de https://www.santafe.gob.ar/ms/ eficienciaenergetica/etiquetado-de-viviendas/pruebas-piloto/

IRAM 11603 - Instituto Argentino de Normalización y Certificación (2012). Acondicionamiento térmico de edificios. Clasificación bioambiental de la República Argentina. Tercera edición.
IRAM 11900 - Instituto Argentino de Normalización y Certificación (2009). Etiqueta de eficiencia energética de calefacción para edificios. Clasificación según la transmitancia térmica de la envolvente.

IRAM 11900 - Instituto Argentino de Normalización y Certificación (2017). Prestaciones energéticas en viviendas, Método de cálculo. Segunda edición.

IRAM 11900 - Instituto Argentino de Normalización y Certificación (2019). Modificación N¹ a la Norma IRAM 11900:2017-12.

Ley 3246 (2009). Consumo de la Energía - Reducción y Optimización. Gobierno de la Ciudad Autónoma de Buenos Aires. 18 de enero de 2010. B.O. No 3342. Recuperado de https://deuco.org.ar/pdf/3246-a.pdf

Ley 4458 (2012). Normas de acondicionamiento térmico en la construcción de edificios. Gobierno de la Ciudad Autónoma de Buenos Aires. 30 de abril de 2012. B.O. No 4142. Recuperado de http://www2.cedom.gob.ar/es/legislacion/normas/leyes/ ley4458.html

Ley 13059 (2003). Condiciones de acondicionamientos térmico exigibles en la construcción de edificios. Gobierno de la Provincia de Buenos Aires. 4 de julio de 2003. B.O. No. 24738. Recuperado de https://normas.gba.gob.ar/ar-b/ley/2003/13059/3792

Ley 13903 (2019). Etiquetado de Eficiencia Energética de inmuebles destinados a vivienda. Gobierno de la Provincia de Santa Fe. 8 de noviembre de 2019. B.O. 21/11/2019. Recuperado de https://www.santafe.gov.ar/normativa/item. php?id=213203\&cod $=d 3275 d 4 f 218763 e 61$ a $255605439155 a 8$

Lizundia, I., Etxepare, L., Sagarna, M. y Uranga, E. J. (2018). El coste de la obligatoria rehabilitación energética de la vivienda colectiva: ¿un problema social? Informes de la Construcción, 70(551), 269. http://dx.doi.org/10.3989/ic.59856

López-Asiain, J., García, M. D. L. N. G., Fernández, C. M., y De Tejada Alonso, A. P. (2020). Influencia de la metodología para la certificación energética de edificios sobre los resultados en el indicador de agua caliente sanitaria. Revista DYNA, 95(3), $257-$ 260. http://dx.doi.org/10.6036/9578

Manzano, F., Montoya, F.G., Sabio-Ortega, A. y García-Cruz, A. (2015). Review of Bioclimatic Architecture Strategies for Achieving Thermal Comfort. Renewable and Sustainable Energy Reviews, 49, 736-755. https://doi.org/10.20868/ade.2019.3913

Martín-Consuegra, F., Oteiza, I., Alonso, C., Cuerdo-Vilches, T. y Frutos, B. (2014). Análisis y propuesta de mejoras para la eficiencia energética del edificio principal del Instituto c.c. Eduardo TorrojaCSIC. Informes de la Construcción, 66(536), e043. http://dx.doi. org/10.3989/ic.14.125.

Ministerio de Energía y Minería (MINEM) (s.f.). Definición y objetivos. Presidencia de la nación. Recuperado de https//www. minem.gob.ar/www/835/26087/definicion-y-objetivos

Ministerio de Desarrollo Productivo (2017). Balance Energético Nacional (BEN) [Archivo Excel]. Argentina.gob.ar. Recuperado de https://www.argentina.gob.ar/produccion/energia/ hidrocarburos/balances-energéticos 
Ministerio de Desarrollo Productivo (s.f.). Etiquetado de Viviendas. Presidencia de la nación. Recuperado de https://www.argentina. gob.ar/produccion/energia/eficiencia-energetica/eficienciaenergetica-en-edificaciones/etiquetado-de-viviendas

Ordenanza 8757 (2011). Aspectos Higrotérmicos y Demanda Energética de las Construcciones. Reglamento de Edificación, modificación. Municipalidad de Rosario. 17 de mayo de 2011. B.O. No 189-2011. Recuperado de https://www.rosario.gob.ar/normativa/ver/visualExterna. do?accion=verNormativa\&idNormativa $=75004$

Pérez Fargallo, A., Calama Rodríguez, J. M. y Flores Alés, V. (2016). Comparativa de resultados de rehabilitación energética para viviendas en función del grado de mejora. Informes de la Construcción, 68(541), 1-11. https://doi.org/10.3989/ic.15.048.

Pérez-Lombard, L, Ortiz, J. R, González, R. y Maes, I.R. (2009). A review of benchmarking, rating and labelling concepts within the framework of building energy certification schemes. Energy Build, 41, 272-278. https://doi.org/10.1016/j.enbuild.2008.10.004

Reus-Netto, G., Mercader-Moyano, P. y Czajkowski, J. D. (2019). Methodological Approach for the Development of a Simplified Residential Building Energy Estimation in Temperate Climate. Sustainability, 11(15), 4040. https://doi.org/10.3390/ su11154040 